\title{
Channel Estimation for PRP-OFDM in Slowly Time-Varying Channel: First-Order or Second-Order Statistics?
}

\author{
Yi Ma, Member, IEEE, Na Yi, Student Member, IEEE, and Rahim Tafazolli
}

\begin{abstract}
The statistics-based channel estimators can estimate the channel state information (CSI) in the static channel but can only obtain the averaged CSI (ACSI) in the time-varying (TV) channel. This letter investigates both first-order statistics (FOS) and second-order statistics (SOS)-based channel estimators for the pseudo random postfix (PRP) orthogonal frequency-division multiplexing system in the slowly TV channel. It is shown that the FOS-based approach outperforms the SOS-based approaches in the ACSI estimation. Using estimated ACSIs for the channel equalization, simulation results indicate that the SOS-based approaches converge to the FOS-based approach in the high signal-to-noise ratio range.
\end{abstract}

Index Terms-Channel estimation, first-order statistics (FOS), orthogonal frequency-division multiplexing (OFDM), second-order statistics (SOS).

\section{INTRODUCTION}

$\mathbf{O}$ RTHOGONAL frequency-division multiplexing (OFDM) modulator usually employs cyclic prefix (CP) or zero padding (ZP) to mitigate the interblock interference (IBI) [1]. These are called CP-OFDM and ZP-OFDM systems, respectively (see [2]). Recent literatures (e.g., [3]) suggested to replace the zero sequence in ZP-OFDM with a number of known symbols and named the proposed system pseudo random postfix (PRP) OFDM. The major advantage of using PRP technique is enabling the low-complexity first-order statistics (FOS)-based estimation of (quasi-) static channels. In the multiple-input multiple-output (MIMO)-OFDM system, employing distinct PRPs at transmit antennas makes the MIMO channel estimation much easier [4].

In the slowly time-varying (TV) channel, statistics-based algorithms can only obtain the averaged channel state information (ACSI). The mismatch between ACSI and instant CSI will increase the equalization error. This motivates us to investigate the ACSI-tracking capability of both FOS-based and SOS-based channel estimation algorithms. To carry out the reasonable comparison, we extended the subspace-based channel estimation algorithm from ZP-OFDM to PRP-OFDM and developed a PRPbased SOS algorithm as well. Pilot symbols are employed to

Manuscript received September 18, 2005; revised November 9, 2005. This work was supported by EU-IST 4More under Project IST-2002-507039. The associate editor coordinating the review of this manuscript and approving it for publication was Dr. Xiang-Gen Xia.

The authors are with Center for Communication Systems Research, University of Surrey, Surrey GU2 7XH, U.K. (e-mail: y.ma@surrey.ac.uk; n.yi@surrey.ac.uk; r.tafazolli@ surrey.ac.uk).

Digital Object Identifier 10.1109/LSP.2005.862620 resolve the residual ambiguity. It is shown that the ACSI obtained by the FOS-based approach is a good estimation of the instant CSIs at the middle of the data record, and the ACSI estimated by the SOS-based approach is a good estimation of instant CSIs at the head of the data record. We observe that the FOS-based approach has stronger capability of tracking the slowly TV channel. Then, the estimated ACSI is used for the channel equalization. Simulation results show that the SOSbased approaches converge to the FOS-based approach in the high signal-to-noise ratio (SNR) range.

\section{PRP-OFDM IN THE TV CHANNEL}

To simplify the presentation and clarify our idea, this letter only considers the single-antenna-based PRP-OFDM system, which is described in [3]. Prior to transmission, the information-bearing blocks $\mathbf{s}_{M}(n)$ with the size $M \times 1$ are fed into ZP-OFDM modulator to generate the ZP-OFDM blocks. The input-output relationship is given by

$$
\mathbf{x}^{(\mathrm{zp})}(n)=\left[\mathbf{F}_{M}, \mathbf{0}_{M \times L_{\mathrm{zp}}}\right]^{\mathcal{H}} \mathbf{s}_{M}(n)
$$

where $\mathbf{F}_{M}$ is the $M \times M$ discrete Fourier transform (DFT) matrix with the $(m, k)$ th entry $\left[\mathbf{F}_{M}\right]_{m, k}=1 / \sqrt{M} \exp (j 2 \pi m k / M), L_{\mathrm{zp}}$ is the length of the zero sequence, and $(\cdot)^{\mathcal{H}}$ is the Hermitian transpose. Then, we let $J=M+L_{\mathrm{zp}}$ and superimpose a training block $\mathbf{p}_{J}=\left[0_{M}^{T}, \mathbf{p}_{L_{\mathrm{zp}}}^{T}\right]^{T}$ onto $\mathbf{x}^{(\mathrm{zp})}(n)$ to produce the PRP-OFDM signal $\mathbf{x}^{(\operatorname{prp})}(n)$ for transmission, i.e.,

$$
\mathbf{x}^{(\operatorname{prp})}(n)=\mathbf{x}^{(\mathrm{zp})}(n)+\mathbf{p}_{J}
$$

where $(\cdot)^{T}$ is the matrix transpose, and $\mathbf{p}_{L_{\mathrm{zp}}}$ is the $L_{\mathrm{zp}} \times 1$ training block. ${ }^{1}$ The PRP-OFDM signal goes through the slowly TV channel with the impulse response $\mathbf{h}(n)=\left[h(n, 0), h(n, 1), \cdots, h\left(n, L_{u}\right)\right]^{T}$, where $L_{u}\left(\leqslant L_{\mathrm{zp}}\right)$ denotes the upper bound of channel order. Due to the slowly TV channel assumption, $\mathbf{h}(n)$ does not vary considerably within a few OFDM blocks [6]. Then, the noiseless version of received blocks are expressible as (see [1] for detail)

$$
\mathbf{y}_{J}(n)=\mathbf{H}^{(1)}(n) \mathbf{x}^{(\mathrm{prp})}(n)+\underbrace{\mathbf{H}^{(\mathrm{u})}(n) \mathbf{x}^{(\mathrm{prp})}(n-1)}_{\text {IBI }}
$$

${ }^{1}$ The reviewer (also in [3]) suggests to use a randomized version of $\mathbf{p}_{J}$ as $\alpha(n) \mathbf{p}_{J}$ to guarantee the spectral flatness, where $\alpha(n)$ is pseudo random with $|\alpha(n)|=1$ and $E\{\alpha(n)\}=0$. Because $\alpha(n)$ is known at the receiver and does not affect the channel estimation (see [3]), we ignore this factor in this letter for convenience. 
where $\mathbf{H}^{(\mathrm{l})}(n)$ and $\mathbf{H}^{(\mathrm{u})}(n)$ are $J \times J$ lower and upper triangular matrices with entries $\left[\mathbf{H}^{(1)}(n)\right]_{m, k}=h(n, m-k)$ and $\left[\mathbf{H}^{(\mathrm{u})}(n)\right]_{m, k}=h(n, J+m-k)$. We plug (2) into (3) and can easily obtain (see [2] for detail)

$$
\mathbf{y}_{J}(n)=\mathbf{C}_{J}(n) \mathbf{x}^{(\mathrm{zp})}(n)+\mathbf{C}_{J}(n) \mathbf{p}_{J}
$$

where $\mathbf{C}_{J}(n)=\mathbf{H}^{(1)}(n)+\mathbf{H}^{(\mathrm{u})}(n)$ is a circulant channel matrix. The linear models (2) and (4) show that the PRP-OFDM is a kind of superimposed-training scheme for the ZP-OFDM system. If the channel is static, then the FOS-based channel estimator can offer very good estimation performance (see [3], [8], and [9]).

Once the channel knowledge $\mathbf{C}_{J}(n)$ is available, the PRP term can be easily eliminated from (4). Then, the residual term becomes the noiseless version of the received ZP-OFDM signal. Equalization of ZP-OFDM has been well addressed in [2]. Later on, we discuss the channel estimation issue based on the noisy version of the received signal, i.e.,

$$
\mathbf{r}_{J}(n)=\mathbf{y}_{J}(n)+\mathbf{v}_{J}(n)
$$

where $\mathbf{v}_{J}(n)$ is the zero-mean white Gaussian noise and is uncorrelated with $\mathbf{y}_{J}(n)$.

\section{Channel Estimation in the Slowly TV ScEnario}

The slowly TV channel is usually modeled by the first-order Markov channel (see [4], [7], and [11]), i.e.,

$$
\mathbf{h}(n)=\mathcal{J}_{0}\left(2 \pi f_{d}\right) \mathbf{h}(n-1)+\boldsymbol{w}(n)
$$

where $\mathcal{J}_{0}(\cdot)$ is the 0 th-order Bessel function of the first kind, $f_{d}$ is the maximum Doppler shift normalized by the OFDM symbol duration, and $\boldsymbol{w}(n)$ is zero-mean Gaussian noise with variance $\sigma_{w}^{2}$. Using the approximation $\mathcal{J}_{0}^{k}\left(2 \pi f_{d}\right) \approx \mathcal{J}_{0}\left(2 \pi k f_{d}\right)$, [4] represents (6) into the following moving average process:

$$
\begin{aligned}
\mathbf{h}(n) & =\sum_{k=0}^{n} \mathcal{J}_{0}\left(2 \pi k f_{d}\right) \boldsymbol{w}(n-k) \\
& =\mathcal{J}_{0}\left(2 \pi n f_{d}\right) \mathbf{h}(0)+\underbrace{\sum_{k=0}^{n-1} \mathcal{J}_{0}\left(2 \pi k f_{d}\right) \boldsymbol{w}(n-k)}_{=\beta(n)}
\end{aligned}
$$

where $\boldsymbol{w}(0)=\mathbf{h}(0)$.

\section{A. FOS-Based Approach}

The FOS-based approach takes the average of $\mathbf{r}_{J}(n)$ to obtain the ACSI. Denoting $N$ to be the data record length (DRL), the average of $\mathbf{r}_{J}(n)$, for $n \in\left[n_{o}, n_{o}+N-1\right]$, can be expressed by

$$
\alpha\left(n_{o}\right)=\frac{1}{N} \sum_{n=0}^{N-1} \mathbf{C}_{J}\left(n_{o}+n\right) \mathbf{p}_{J}+\overline{\mathbf{v}}_{J}\left(n_{o}\right)
$$

where $n_{o}$ denotes the start index, and $\overline{\mathbf{v}}_{J}\left(n_{o}\right)$ is given by

$$
\overline{\mathbf{v}}_{J}\left(n_{o}\right)=\frac{1}{N} \sum_{n=0}^{N-1}\left(\mathbf{C}_{J}\left(n_{o}+n\right) \mathbf{x}^{(\mathrm{zp})}\left(n_{o}+n\right)+\mathbf{v}_{J}\left(n_{o}+n\right)\right) \text {. }
$$

Then, we perform $J$-point DFT on $\alpha\left(n_{o}\right)$ and obtain (see [2] for detail derivation)

$$
\begin{aligned}
\tilde{\alpha}\left(n_{o}\right) & =\mathbf{F}_{J} \alpha\left(n_{o}\right) \\
& =\underbrace{\mathbf{D}\left(\mathbf{F}_{J} \mathbf{p}_{J}\right) \overline{\mathbf{F}}_{J}}_{\mathbf{G}}\left(\frac{1}{N} \sum_{n=0}^{N-1} \mathbf{h}\left(n_{o}+n\right)\right)+\tilde{\mathbf{v}}_{J}\left(n_{o}\right)
\end{aligned}
$$

where $\tilde{\mathbf{v}}_{J}\left(n_{o}\right)=\mathbf{F}_{J} \overline{\mathbf{v}}_{J}\left(n_{o}\right), \overline{\mathbf{F}}_{J}$ is formed by the first $\left(L_{u}+\right.$ 1) columns of the $J$-point DFT matrix $\mathbf{F}_{J}$, and $\mathbf{D}(\mathbf{a})$ denotes a diagonal matrix with the vector $\mathbf{a}$ in its diagonal. If $\mathbf{G}$ has the full-column rank, [3] uses $\hat{\mathbf{h}}\left(n_{o}\right)=\mathbf{G}^{\dagger} \tilde{\alpha}\left(n_{o}\right)$ to obtain the channel estimate in the static channel, where $(\cdot)^{\dagger}$ denotes the pseudo inverse. In the slowly TV channel, $\hat{\mathbf{h}}\left(n_{o}\right)$ is the estimated ACSI given by

$$
\hat{\mathbf{h}}\left(n_{o}\right)=\frac{1}{N} \sum_{n=0}^{N-1} \mathbf{h}\left(n_{o}+n\right)+\mathbf{G}^{\dagger} \tilde{\overline{\mathbf{v}}}_{J}\left(n_{o}\right) .
$$

Using (7), we are able to rewrite (11) into the following form:

$$
\hat{\mathbf{h}}\left(n_{o}\right)=\frac{1}{N} \sum_{n=0}^{N-1} \mathcal{J}_{0}\left(2 \pi n f_{d}\right) \mathbf{h}\left(n_{o}\right)+\bar{\beta}\left(n_{o}\right)+\mathbf{G}^{\dagger} \tilde{\overline{\mathbf{v}}}_{J}\left(n_{o}\right)
$$

where $\bar{\beta}\left(n_{o}\right)$ is the average of $\beta(n)$. Since $N f_{d}$ is practically small (e.g., 0.1$)$ in the slowly TV scenario, $\mathcal{J}_{0}\left(2 \pi n f_{d}\right)$ is a monotonically decreasing (increasing) function for $n \in[0, N-$ $1]$. Then, we can find an index $n_{\xi} \in[0, N-1]$, which fulfills

$$
\mathcal{J}_{0}\left(2 \pi n_{\xi} f_{d}\right) \cong \frac{1}{N} \sum_{n=0}^{N-1} \mathcal{J}_{0}\left(2 \pi n f_{d}\right) .
$$

Plugging (13) into (12), we have

$$
\begin{aligned}
& \hat{\mathbf{h}}\left(n_{o}\right) \cong \mathcal{J}_{0}\left(2 \pi n_{\xi} f_{d}\right) \mathbf{h}\left(n_{o}\right)+\bar{\beta}\left(n_{o}\right)+\mathbf{G}^{\dagger} \tilde{\overline{\mathbf{v}}}_{J}\left(n_{o}\right) \\
& =\mathbf{h}\left(n_{o}+n_{\xi}\right)+\underbrace{\bar{\beta}\left(n_{o}\right)-\beta\left(n_{o}+n_{\xi}\right)+\mathbf{G}^{\dagger} \tilde{\overline{\mathbf{v}}}_{J}\left(n_{o}\right)}_{\text {interference }} .
\end{aligned}
$$

This result shows that the estimated ACSI is also the estimate of the instant CSI at the block index $\left(n_{o}+n_{\xi}\right)$. Moreover, we observe that $\mathcal{J}_{0}\left(2 \pi n f_{d}\right)$ approximates a first-order polynomial for $n \in[0, N-1]$; therefore, $n_{\xi}$ should be close to $N / 2$.

\section{B. Subspace-Based Approach}

The subspace-based approach is based on the autocorrelation of $\mathbf{r}_{J}(n)$, which is practically implemented by

$$
\begin{aligned}
\mathcal{C}\left(n_{o}, n_{\tau}\right) & =\frac{1}{N-n_{\tau}} \sum_{n=n_{\tau}}^{N-1} \mathbf{r}_{J}\left(n_{o}+n\right) \mathbf{r}_{J}^{\mathcal{H}}\left(n_{o}+n+n_{\tau}\right) \\
& \approx \mathcal{C}^{(s)}\left(n_{o}, n_{\tau}\right)+\mathcal{C}^{(p)}\left(n_{o}, n_{\tau}\right)+\mathcal{C}^{(v)}\left(n_{\tau}\right)
\end{aligned}
$$

for

$$
\begin{aligned}
\mathcal{C}^{(s)}\left(n_{o}, n_{\tau}\right) & =\sigma_{s}^{2} E\left\{\overline{\mathbf{C}}_{J}\left(n_{o}\right) \overline{\mathbf{C}}_{J}^{\mathcal{H}}\left(n_{o}+n_{\tau}\right)\right\} \delta\left(n_{\tau}\right) \\
\mathcal{C}^{(p)}\left(n_{o}, n_{\tau}\right) & =E\left\{\mathbf{C}_{J}\left(n_{o}\right) \mathbf{p}_{J} \mathbf{p}_{J}^{\mathcal{H}} \mathbf{C}_{J}^{\mathcal{H}}\left(n_{o}+n_{\tau}\right)\right\} \\
\mathcal{C}^{(v)}\left(n_{\tau}\right) & =\sigma_{v}^{2} \mathbf{I}_{J} \delta\left(n_{\tau}\right)
\end{aligned}
$$


where $\sigma_{s}^{2}$ and $\sigma_{v}^{2}$ denote the variances of signal and noise, respectively, $\overline{\mathbf{C}}_{J}(n)$ is formed by the first $\left(J-L_{\text {zp }}\right)$ columns of $\mathbf{C}_{J}(n)$, and $\delta(\cdot)$ is the Dirac delta function. References [11] and [12] have indicated that autocorrelation of the slowly TV channel is

$$
E\left\{\mathbf{h}\left(n_{o}\right) \mathbf{h}^{\mathcal{H}}\left(n_{o}+n_{\tau}\right)\right\}=\mathcal{J}_{0}\left(2 \pi n_{\tau} f_{d}\right) \mathbf{h}\left(n_{o}\right) \mathbf{h}^{\mathcal{H}}\left(n_{o}\right) .
$$

Equivalently, the following result holds:

$$
E\left\{\overline{\mathbf{C}}_{J}\left(n_{o}\right) \overline{\mathbf{C}}_{J}^{\mathcal{H}}\left(n_{o}+n_{\tau}\right)\right\}=\mathcal{J}_{0}\left(2 \pi n_{\tau} f_{d}\right) \overline{\mathbf{C}}_{J}\left(n_{o}\right) \overline{\mathbf{C}}_{J}^{\mathcal{H}}\left(n_{o}\right) .
$$

Applying this result into (17), we can conclude [see (24) and (25)]

$$
\mathcal{C}^{(p)}\left(n_{o}, 1\right)=\underbrace{\mathcal{J}_{0}\left(2 \pi f_{d}\right)}_{=1} \mathcal{C}^{(p)}\left(n_{o}, 0\right) .
$$

Because $\mathcal{C}\left(n_{o}, n_{\tau}\right)=\mathcal{C}^{(p)}\left(n_{o}, n_{\tau}\right)$ for $n_{\tau} \neq 0$, we are able to remove the term $\mathcal{C}^{(p)}\left(n_{o}, 0\right)$ from $\mathcal{C}\left(n_{o}, 0\right)$ using

$$
\begin{aligned}
\Delta\left(n_{o}\right) & =\mathcal{C}\left(n_{o}, 0\right)-\mathcal{C}\left(n_{o}, 1\right) \\
& =\mathcal{C}^{(s)}\left(n_{o}, 0\right)+\sigma_{v}^{2} \mathbf{I}_{J} .
\end{aligned}
$$

We then plug (20) into (22) and obtain

$$
\Delta\left(n_{o}\right)=\sigma_{s}^{2} \overline{\mathbf{C}}_{J}\left(n_{o}\right) \overline{\mathbf{C}}_{J}^{\mathcal{H}}\left(n_{o}\right)+\sigma_{v}^{2} \mathbf{I}_{J} .
$$

Based on (23), [5] used singular value decomposition (SVD) to estimate $\mathbf{h}\left(n_{o}\right)$, regardless of channel nulls (up to a scalar ambiguity). We can see that the ACSI obtained by the subspacebased approach is the estimate of the instant CSI at the block index $n_{o}$. In order to resolve the residual scalar ambiguity, pilot symbols should be placed in the $n_{o}$ th OFDM block.

\section{PRP-Based SOS Approach}

In the subspace-based approach, $\mathcal{C}^{(p)}\left(n_{o}, n_{\tau}\right)$ is treated as the interference term and needs to be removed. Actually, $\mathcal{C}^{(p)}\left(n_{o}, n_{\tau}\right)$ can also be used to obtain the ACSI. Reconsidering $\mathcal{C}\left(n_{o}, n_{\tau}\right)$ for $n_{\tau}=1$, we have

$$
\mathcal{C}\left(n_{o}, 1\right)=\underbrace{E\left\{\mathbf{C}_{J}\left(n_{o}\right) \mathbf{p}_{J} \mathbf{p}_{J}^{\mathcal{H}} \mathbf{C}_{J}^{\mathcal{H}}\left(n_{o}+1\right)\right\}}_{=\mathcal{C}^{(p)}\left(n_{o}, 1\right)} .
$$

Using the result $\mathbf{C}_{J}\left(n_{o}\right)=\mathbf{F}_{J}^{\mathcal{H}} \mathbf{D}\left(\overline{\mathbf{F}}_{J} \mathbf{h}\left(n_{o}\right)\right) \mathbf{F}_{J}$ (see [1] and [10]), (24) can be represented as

$$
\begin{aligned}
\mathcal{C}\left(n_{o}, 1\right) & =\mathbf{F}_{J}^{\mathcal{H}} \mathbf{G} E\left\{\mathbf{h}\left(n_{o}\right) \mathbf{h}^{\mathcal{H}}\left(n_{o}+1\right)\right\} \mathbf{G}^{\mathcal{H}} \mathbf{F}_{J} \\
& =\underbrace{\mathcal{J}_{0}\left(2 \pi f_{d}\right)}_{=1} \underbrace{\mathbf{F}_{J}^{\mathcal{H}} \mathbf{G h}\left(n_{o}\right) \mathbf{h}^{\mathcal{H}}\left(n_{o}\right) \mathbf{G}^{\mathcal{H}} \mathbf{F}_{J}}_{=\mathcal{C}^{(p)}\left(n_{o}, 0\right)} .
\end{aligned}
$$

Then, we can easily obtain

$$
\mathbf{h}\left(n_{o}\right) \mathbf{h}^{\mathcal{H}}\left(n_{o}\right)=\mathbf{G}^{\dagger} \mathbf{F}_{J} \mathcal{C}\left(n_{o}, 1\right) \mathbf{F}_{J}^{\mathcal{H}}\left(\mathbf{G}^{\dagger}\right)^{\mathcal{H}}
$$

and obtain $\mathbf{h}\left(n_{o}\right)$ from (26) (up to a phase ambiguity). Compared with the subspace-based approach, the PRP-based approach does not need SVD and thus reduces the computation complexity.

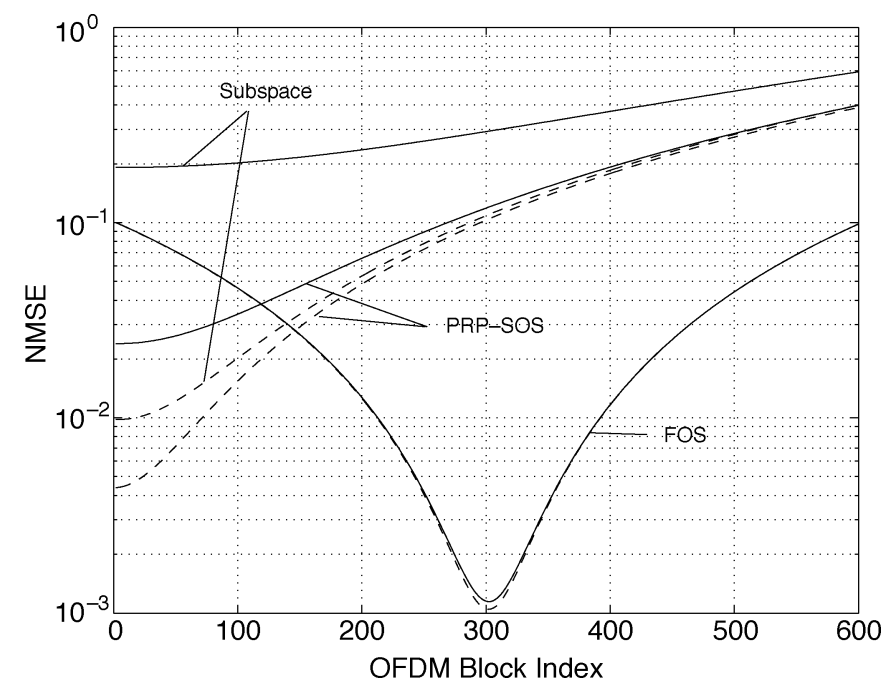

Fig. 1. Channel NMSE along the block index with the terminal speed of $v=$ $6 \mathrm{~m} / \mathrm{s}, \mathrm{SNR}=12 \mathrm{~dB}$ for solid lines, $20 \mathrm{~dB}$ for dash lines.

\section{Simulation Results}

To benchmark the channel estimators, we used the normalized mean-square-error (NMSE) of the channels defined as $1 / \mathcal{I} \sum_{i=0}^{\mathcal{I}-1}\left\|\hat{\mathbf{h}}_{i}-\mathbf{h}_{i}\right\|^{2} /\left\|\mathbf{h}_{i}\right\|^{2}$. Here, $\mathcal{I}$ is the number of Monte Carlo trials. The parameters of PRP-OFDM systems were set as $M=32, L_{\mathrm{zp}}=4$, and $J=M+L_{\mathrm{zp}}=36$. The information-bearing symbols were drawn from the quadrature phase-shift keying (QPSK) constellation with the equal probability. The subcarrier spacing $\Delta f$ of $312.5 \mathrm{KHz}$ was the same as HIPERLAN/2 [13]. The PRP was constructed by the Kaiser window [14], which was the same as the setting in [3]. The PRP-to-information power ratio was given by $L_{\mathrm{zp}} / M$. The SNR is defined by the average received bit energy to noise $E_{b} / \mathcal{N}_{o}$ [11]. The slowly TV finite impulse response (FIR) channels are generated according to the channel model A specified by ETSI for HIPERLAN/2 [15], where each tap varies according to Jakes' model with a maximum Doppler frequency of $104 \mathrm{~Hz}$ corresponding to the terminal speed $v=6 \mathrm{~m} / \mathrm{s}$ and an operating frequency of $5.2 \mathrm{GHz}$.

1) Evaluation of Channel NMSE: The channel estimation was implemented by collecting $N=300$ PRP-OFDM blocks. We averaged simulation results over $\mathcal{I}=500$ Monte Carlo trials. Fig. 1 shows the NMSE for the investigated channel estimators at SNR $=12 \mathrm{~dB}$ (typical SNR) and $20 \mathrm{~dB}$, respectively. It can be observed that the FOS-based estimator is not sensitive to the SNR. Consistent with the conclusion in Section III-A, Fig. 1 shows that the FOS-based approach can estimate the instant CSI accurately at the block index $n_{\xi} \approx N / 2$. However, the NMSE increases rapidly with the increase of $\left|n-n_{\xi}\right|$. Note that the subspace-based approach uses six pilot symbols in the first OFDM block to resolve the scalar ambiguity. Its channel NMSE is related to the estimation accuracy of the residual scalar, which is quite sensitive to the SNR. The PRP-based SOS estimator only needs to resolve a phase ambiguity and thus outperforms the subspace-based estimator.

2) Evaluation of Overall System Performance: We assume that each data burst (a frame) has 600 PRP-OFDM blocks. To resolve the residual ambiguity, six pilot symbols are embedded 


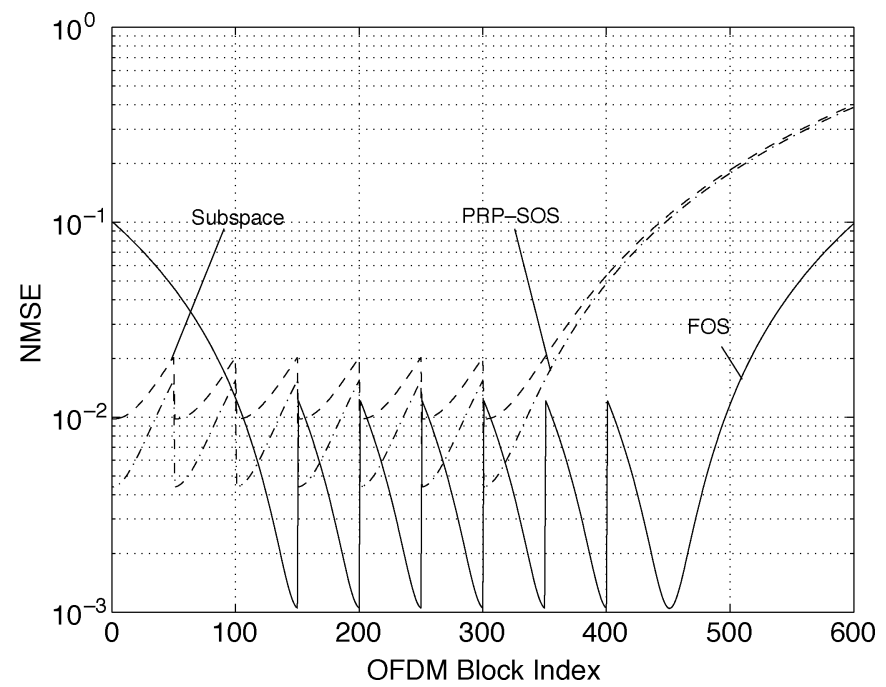

Fig. 2. Channel NMSE along the data burst with the terminal speed $v=6 \mathrm{~m} / \mathrm{s}$, $\mathrm{SNR}=20 \mathrm{~dB}$.

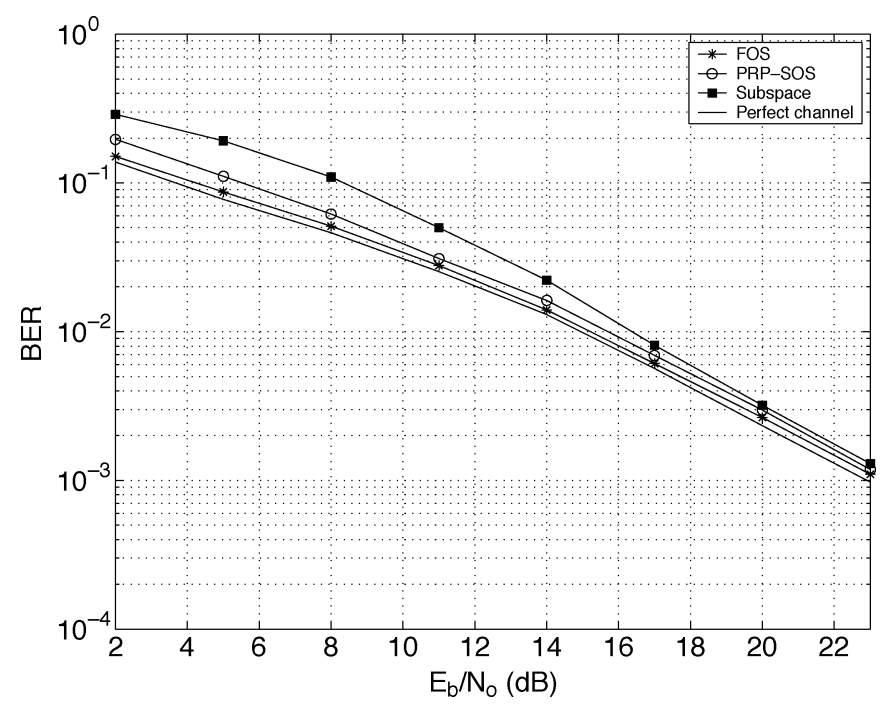

Fig. 3. BER versus SNR for the terminal speed $v=6 \mathrm{~m} / \mathrm{s}$.

for every 50 PRP-OFDM blocks. The channel tracking method is given as follows.

1) Collect the first $N=300$ blocks in a frame for the channel estimation.

2) Each new channel estimation is implemented by discarding the first 50 old data records and collecting 50 new blocks.

Using the above channel tracking method, the estimation performance along the data burst is plotted in Fig. 2. Fig. 3 shows bit-error rate (BER) as a function of SNR. We can see that the FOS-based approach dominates the SOS-based approaches. Their performance is very close in the high-SNR range. We can also observe that the PRP-based SOS approach outperforms the subspace-based approach.

\section{CONCLUSION}

In this letter, we have investigated the FOS- and SOS-based channel estimators for PRP-OFDM in the slowly TV channel. Theoretical analysis together with simulation results have shown that the FOS-based approach has stronger capability of tracking the slowly TV channel, particularly in the low and moderate SNR range.

\section{REFERENCES}

[1] Z. Wang and G. B. Giannakis, "Wireless multicarrier communications: Where Fourier meets Shannon," IEEE Signal Process. Mag., vol. 17, no. 3, pp. 29-48, May 2000.

[2] B. Muquet, Z. Wang, G. B. Giannakis, M. Courville, and P. Duhamel, "Cyclic prefixing or zero padding for wireless multicarrier transmissions," IEEE Trans. Commun., vol. 50, no. 12, pp. 2136-2148, Dec. 2002.

[3] M. Muck, M. de Courville, M. Debbah, and P. Duhamel, "A pseudo random postfix OFDM modulator and inherent channel estimation techniques," in Proc. IEEE Global Telecommunications Conf., vol. 4, Dec. 2003, pp. 2380-2384.

[4] M. Muck, A. R. Dias, M. de Courville, and P. Duhamel, "A pseudo random postfix OFDM based modulator for multiple antennae systems," in Proc. IEEE Int. Conf. Communications, vol. 4, Jun. 2004, pp. 2392-2396.

[5] B. Muquet, M. de Courville, and P. Duhamel, "Subspace-based blind and semi-blind channel estimation for OFDM systems," IEEE Trans. Signal Process., vol. 50, no. 7, pp. 1699-1712, Jul. 2002.

[6] T. S. Rappaport, Wireless Communications: Principles and Practice. Englewood Cliffs, NJ: Prentice-Hall, 1996.

[7] R. Negi and J. Cioffi, "Pilot tone selection for channel estimation in a mobile OFDM system," IEEE Trans. Consum. Electron., vol. 44, no. 3, pp. 1122-1128, Aug. 1998.

[8] F. Mazzenga, "Channel estimation and equalization for M-QAM transmission with a hidden pilot sequence," IEEE Trans. Broadcast., vol. 46, no. 2, pp. 170-176, Jun. 2000.

[9] J. K. Tugnait and W. Luo, "On channel estimation using superimposed training and first-order statistics," IEEE Commun. Lett., vol. 7, no. 9, pp. 413-415, Sep. 2003.

[10] G. H. Golub and C. F. Van Loan, Matrix Computations, 3rd ed. Baltimore, MD: The Johns Hopkins Univ. Press, 1996.

[11] J. Proakis, Digital Communications, 4th ed. New York: McGraw-Hill, 2000.

[12] Y. Li, L. J. Cimini Jr., and N. Scollenberger, "Robust channel estimation for OFDM systems with rapid dispersive fading channels," IEEE Trans. Commun., vol. 46, no. 7, pp. 902-915, Jul. 1998.

[13] ETSI, Broadband Radio Access Networks (BRAN); High Performance Radio Local Area Networks (HIPERLAN) Type 2: Physical Layer, Document DTS0023003, France, Dec. 1999.

[14] A. V. Oppenheim and R. W. Schafer, Discrete-Time Signal Processing. Englewood Cliffs, NJ: Prentice-Hall, 1989.

[15] J. Medbo and P. Schramm, Channel models for HIPERLAN/2 in different indoor scenarios, in BRAN 3ERI085B, Mar. 1998. 